\title{
Hernies Ombilicales Étranglées De L'enfant Au Centre Hospitalier Régional De Ziguinchor (Sénégal)
}

\section{Diouf Cheikh}

Département de chirurgie et spécialités chirurgicales. Centre hospitalier régional de Ziguinchor. Université Assane Seck de Ziguinchor Sénégal

\section{Sow Omar \\ Diallo Ibrahima}

Centre hospitalier régional de Ziguinchor Sénégal

Ndoye Ndeye Aby

Département de chirurgie et spécialités chirurgicales. Centre hospitalier national d'enfants Albert Royer. Université cheikh Anta Diop Dakar Sénégal

\section{Ngom Gabriel}

Département de chirurgie et spécialités chirurgicales. Hôpital Aristide Le

Dantec. Université cheikh Anta Diop Dakar Sénégal

Doi: 10.19044/esj.2017.v13n36p379 URL:http://dx.doi.org/10.19044/esj.2017.v13n36p379

\begin{abstract}
The umbilical hernia is a well-known pathology even though, its strangulation has been subject to much controversy. The aim of this work is to report our experience in the management of strangulated hernia of the child at the Ziguinchor regional hospital and to compare our results with those of the literature through a retrospective and descriptive study over a four years period. During the study period 24 patients with high male predominance were received. The mean age of the patients was 2.1 years. $45 \%$ of patients had a chronic cough. The average time for consultation was 27.6 hours. A notion of abdominal abrupt was found in $45 \%$ of the cases. The vast majority of patients arrived at the hospital during the dry season. Abdominal pain and vomiting were the main symptoms. An occlusive syndrome was present in about half of the patients. The diameter of the collar of the hernia varied between 1.5 and $2.5 \mathrm{~cm}$ and no intestinal necrosis was found during exploration. The surgery was simple in the majority of cases. Umbilical hernia is a reality in under-developed environment some factors promotes its complications.
\end{abstract}

Keywords: Umbilical hernia, strangulated hernia, bowel obstruction, children 


\section{Résumé}

La hernie ombilicale est une pathologie bien connue même si son étranglement a été sujet à beaucoup de controverses. Le but de ce travail est de rapporter notre expérience dans la prise en charge de la hernie étranglée de l'enfant au centre hospitalier régional de Ziguinchor à travers une étude rétrospective de type descriptive sur une période de quatre ans. Durant la période d'étude 24 patients dont 15 garçons et 09 filles ont été reçus. L'âge moyen des patients était de 2,1 ans. $45 \%$ des patients présentaient une toux chronique. Le délai moyen de consultation était de 27,6 heures. Une notion de douleur abdominale brutale a été retrouvée dans $45 \%$ des cas. $83,33 \%$ patients était arrivée à l'hôpital pendant la saison sèche. La douleur abdominale et les vomissements étaient les maitres symptômes. Un syndrome occlusif était présent dans un peu prés de la moitié des patients. Le diamètre du collet de la hernie variait entre 1,5 et $2,5 \mathrm{~cm}$ et aucune nécrose intestinale n'a été retrouvée à l'exploration. Les suites opératoires ont été simples dans $83,4 \%$ des cas. La hernie ombilicale est une réalité en milieu sous développé certains facteurs favorisent des complications

Mots clés : Hernie ombilicale, étranglement herniaire, occlusion intestinale, enfants

\section{Introduction}

La hernie ombilicale est due à un retard de fermeture de l'anneau ombilical. Il existe des facteurs favorisant ce défaut de fermeture tels que l'hypotrophie, l'hérédité, l'hyperpression intra-abdominale (Weik, 2005). Même si au plan épidémiologique sa fréquence est bien connue, celle de ses complications notamment l'étranglement est encore sujet à beaucoup de controverses. En Afrique et en Asie, ses complications surtout l'étranglement sont bien connues alors qu'en Europe et aux Etats Unis d'Amérique, plusieurs auteurs défendent encore l'hypothèse de la rareté de cet étranglement (Stovroff, 2001) (Ameh, 2003) (Ngom, 2015) (Koura, 1996) (Brown, 2006) . Le but de notre étude était de rapporter les aspects épidémiologiques, cliniques, thérapeutiques et évolutifs des hernies ombilicales étranglées de l'enfant au Centre Hospitalier Régional de Ziguinchor (CHRZ), qui est l'hôpital de référence de la région sud du Sénégal. 


\section{Matériel et méthodes}

Il s'agit d'une étude rétrospective de type descriptif sur une période de 4 ans ( $1^{\text {er }}$ juin 2012 au 31 mai 2016). Elle a porté sur les dossiers des enfants âgés de moins de 16 ans, hospitalisés dans le service de chirurgie générale du CHRZ pour hernie ombilicale étranglée. Le diagnostic de hernie ombilicale étranglée était posé devant une tuméfaction ombilicale douloureuse et irréductible d'apparition brutale chez un enfant ayant ou non des antécédents connus de hernie ombilicale. Cette tuméfaction ombilicale pouvant être associée à des vomissements ou à un arrêt du transit intestinal. Les paramètres épidémiologiques (fréquence, âge, sexe, antécédents), cliniques (circonstances de découvertes, examen physique) thérapeutiques (traitements institués et évolutifs (évolution à court moyen et long terme) étaient étudiés.

\section{Résultats}

\section{Aspects épidémiologiques}

Durant la période d'étude, 1762 patients âgés de moins de 16 ans étaient admis dans le service de chirurgie, dont 140 pour hernie ombilicale soit une fréquence globale de 7,94\%. Parmi ces 140 patients, 24 patients $(17,14 \%$ ) avaient un étranglement herniaire. L'âge moyen des patients était de 2,1 ans avec des extrêmes de 1 mois et 13 ans. Il y avait 15 garçons et 9 filles; le sexe ratio était de 1,55. Huit patients avaient un âge compris entre 1 mois et 11 mois et les autres un âge compris entre 12 mois et 13 ans. Quinze patients étaient suivis dans le service pour hernie ombilicale en attente d'intervention chirurgicale. Aucun cas d'antécédent familial de hernie ombilicale n'avait été noté. La Répartition des patients suivant la période d'admission est présentée dans le tableau I. Une notion de toux était rapportée chez --- patients (45\%). Aucun antécédent d'engouement herniaire n'avait été signalé. Le délai moyen de consultation aux urgences du CHRZ était de 27,6 heures, avec des extrêmes de 10 heures et 48 heures.

Tableau 1 : Répartition des patients suivant la période d'admission (les saisons).

\begin{tabular}{ccc}
\hline Saisons & $\begin{array}{c}\text { Nombre de } \\
\text { patients }\end{array}$ & Proportion en \% \\
\hline Saison sèche (octobre - juin) & 20 & 83,33 \\
Saison pluvieuse (juillet - septembre) & 4 & 16,67 \\
\hline Total & $\mathbf{2 4}$ & $\mathbf{1 0 0}$ \\
\hline
\end{tabular}




\section{Aspects cliniques}

La douleur abdominale et la tuméfaction ombilicaleétaient les principaux symptômes, retrouvés chez tous les patients. Elles étaient associées à des vomissements dans $65 \%$ des cas. Un syndrome occlusif était retrouvé chez $48 \%$ des patients. Un patient présentait une hernie ombilicale étranglée associée à un suintement séreux de l'ombilic.

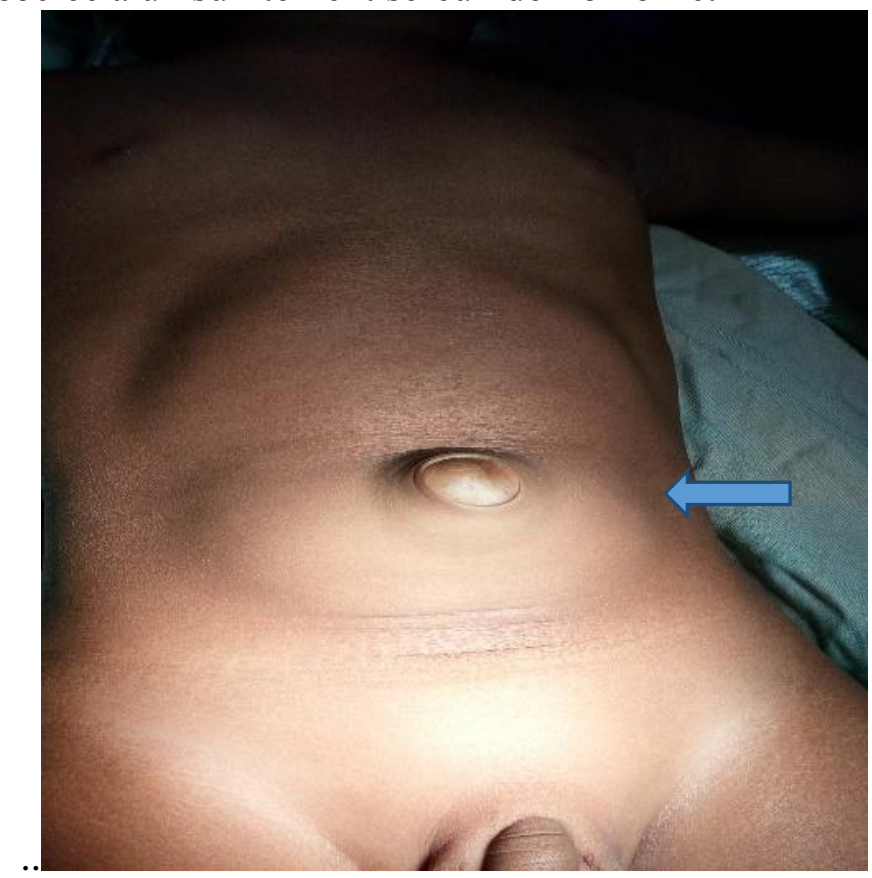

Figure 1 : Tuméfaction ombilicale chez un enfant présentant une hernie ombilicale étranglée. Peau ombilicale tendue et luisante

\section{Aspects thérapeutiques}

Après une réanimation intensive, les patients avaient tous bénéficié, sous anesthésie générale, d'une intervention chirurgicale pour lever l'étranglement, et traiter la hernie et son contenu. Le diamètre du collet de la hernie après incision exploration et réduction était en moyenne de $1,9 \mathrm{~cm}$ avec des extrêmes de 1,5 et $2,5 \mathrm{~cm}$ chez tous nos patients. La voie d'abord était une incision arciforme sous ombilicale. L'exploration chirurgicale après ouverture du sac herniaire retrouvait une anse grêle chez 17 patients. Parmi ces 17 patients on a remarqué un contenu intestinal épais dans l'anse grêle chez 6 patients. L'anse intestinale contenue dans la hernie était viable dans tous les cas (figure 2). Le sac herniaire contenait la jonction iléo-cæcale chez 2 patients et le grand omentum chez 5 patients. Un patient présentait un kyste de l'ouraque fistulisé à l'ombilic associé. Une cure par aponévrorraphie fut réalisée chez tous les patients. La durée moyenne d'hospitalisation était de 2,5 jours, avec des extrêmes de 2 et 4 jours. 


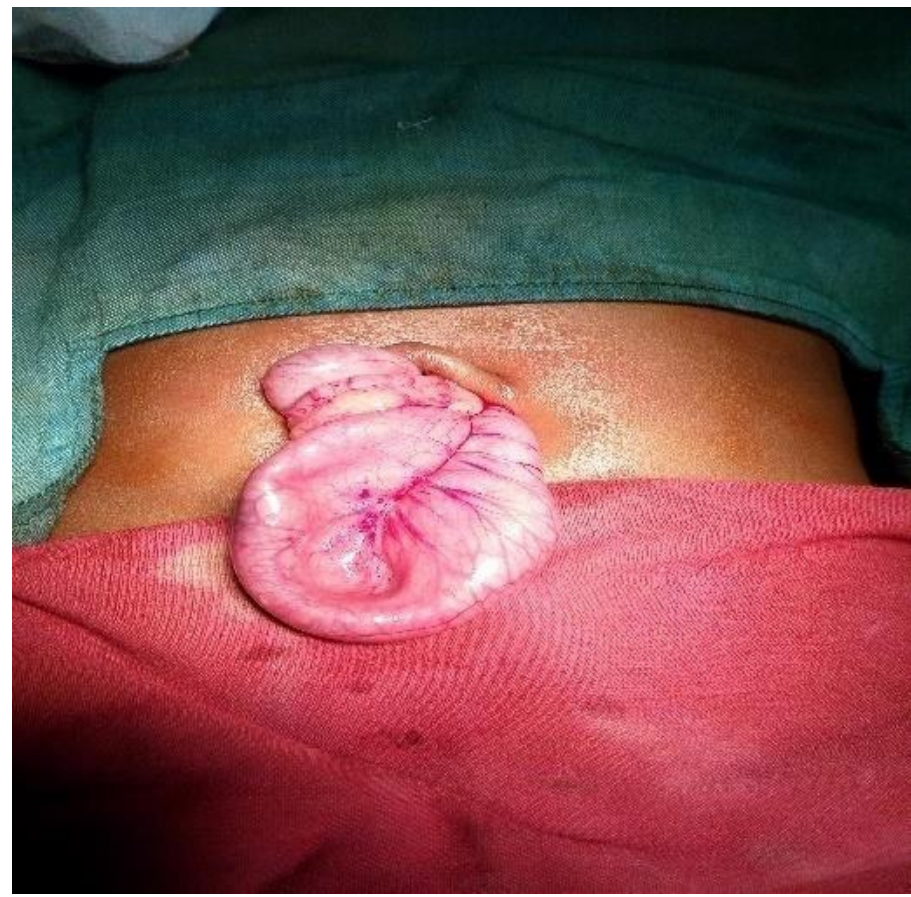

Figure 2: Anse grêle viable après exploration chirurgicale

\section{Aspects évolutifs}

Dans les suites opératoires, une suppuration pariétale était retrouvée chez 4 patients soit 16,6 \% . Avec un recul de moyen de 10,2 mois (extrêmes 5 mois et 4,4 ans) aucune récidive n'avait été notée.

\section{Discussion}

\section{Aspects épidémiologiques}

La hernie ombilicale de l'enfant est une affection fréquente et réputée bénigne [4]. Elle est due à un retard de fermeture de l'anneau ombilical associé à des facteurs favorisants tels que : hypotrophie, hérédité, hyperpression intra-abdominale (Weik 2005). Généralement asymptomatique, l'évolution s'effectue vers une fermeture spontanée de l'anneau ombilical dans les trois premières années de vie. Contrairement aux hernies inguinales, le risque d'étranglement est minime (Koura, 1996). Dans les pays développés l'étranglement de la hernie ombilicale est rarement rapporté dans la littérature, alors qu'en Afrique noire, le nombre de cas rapportés ne cesse d'augmenter (Ameh , 2003) (Ngom, 2015) (Koura, 1996) (Brown, 2006) ; il en est de même en Inde ( Lassaleta, 1975) (Fall 2006). Sa fréquence hospitalière estimée à 7,94\% dans notre étude varie entre 5 et 20 $\%$ selon les auteurs (Khen-dunlop, 2007). A Dakar, Mawukpe et al, ( Mawukpe,1971) en avaient trouvé 20 cas en 8 ans 9 mois (1963-1971). En Inde, Harbans et al. ( Harbans, 1972) ont constaté sur une série de 1600 
hernies chez l'enfant, que $24,15 \%$ des hernies ombilicales étaient étranglées. Par contre les travaux effectués en Europe et en Amérique ne signalent que quelques rares cas d'étranglement de hernies ombilicales (Miller, 1932) (Mestel,1963) (Ndoma, 2013) (Bandré 2010) (Angate, 1972) (Liberek, 2013) . Au Massachussetts, Miller (Miller, 1932) n'a relevé qu'un seul cas en 20 ans. A New York, Mestel (Mestel,1963) n'a trouvé qu'un seul cas d'étranglement sur 1500 hernies ombilicales vues en consultation. Pour certains auteurs aux Etats Unis d'Amérique, la fréquence des étranglements est estimée à 1\% (Miller, 1932) (Mestel,1963) (Ndoma,2013) (Bandré, 2010) (Angate, 1972) (Liberek, 2013). En définitive la rareté de l'étranglement herniaire ombilical n'apparaît que dans les pays développés (Liberek, 2013) (Ngom,2016). Des facteurs favorisant ont été incriminés dans le processus d'étranglement des hernies ombilicales. Ainsi Fall (Fall, 2006) défend la thèse d'un corps étranger favorisant l'étranglement alors que Ngom (Ngom,2016) pense à une influence saisonnière ou une notion d'hyperpression abdominale. Dans notre série $45 \%$ de nos patients présentaient une notion de toux chronique; dans la série de Ngom (Ngom,2016), 65\% de ses patients avaient soit une rhinite, un asthme ou une pneumonie. L'influence saisonnière défendue par Ngom et al au Sénégal pourrait être une hypothèse valable dans notre contexte où $83,33 \%$ de nos patients ont été reçus durant la saison sèche $($ Ngom,2016) . En effet cette période est caractérisée par une alternance parfois brutale de chaleur intense et de vents frais et poussiéreux faisant chuter brutalement les températures (décembre janvier février) (Ngom,2016) ]. L'ensemble de ces phénomènes pourrait expliquer la fréquence accrue des pneumopathies, des crises d'asthme qui créent une hyperpression abdominale brutale et favorisent par la même occasion l'étranglement de la hernie ombilicale (Ngom,2016) .

L'âge moyen de nos patients de 2,1 ans concorde avec beaucoup de données de la littérature (Ngom, 2015) Koura 1996) (Miller 1932) Mestel 1963). La hernie ombilicale est une pathologie nettement plus fréquente chez le garçon comme retrouve dans notre étude et par d'autres auteurs (Ngom, 2015) Koura 1996) (Miller 1932) (Mestel 1963).

\section{Aspects cliniques}

Le délai moyen de consultation aux urgences chez nos patients qui était de 27,6 heures est comparable à celui rapporté par Ngom (Ngom, 2015) [4] et Koura (Koura 1996) . Ainsi la proportion de patients admis dans les 24h suivant l'étranglement était de 78,41 \% à Cotonou (Koura 1996) et de $57,1 \%$ pour Ngom à Dakar (Ngom, 2015). La tuméfaction ombilicale douloureuse reste la symptomatologie commune des hernies ombilicales étranglées. Dans l'étude de Ngom elle est présente dans 97,1\% des cas (Ngom, 2015). Elle était associée à des vomissements dans 65\% des cas dans 
notre travail alors que dans l'étude de Ngom les vomissements étaient présents dans 62,8\% des cas (Ngom, 2015). Un syndrome occlusif a été retrouvé dans $48 \%$ des patients dans notre travail alors que pour Fall (Fall 2006), Ngom (Ngom, 2015) [4], et Bandré (Bandré,2010), la fréquence de ce syndrome occlusif était respectivement de $41,4 \%, 11,4 \%$ et $80 \%$. Les douleurs abdominales en cas d'étranglement de hernie ombilicale sont fonction du contenu et de la durée de l'étranglement. En effet, les épiplocèle et les pincements latéraux sont plus douloureuses (Wade, 2015) John, 2011). La présence du syndrome occlusif serait étroitement liée à la durée de l'étranglement, au contenu du sac herniaire (anse grêle ou caecum), et au diamètre du collet de la hernie.

\section{Aspects thérapeutiques}

Le diamètre du collet constitue un facteur important dans la prise en charge des hernies car il est inversement proportionnel au risque d'étranglement (Koura, 1996) (Ngom, 2016) (Bandré 2010).

L'intestin grêle était l'organe le plus retrouvé dans le sac herniaire dans notre étude et dans celle de Bandré (Bandré 2010). Par contre, pour Angate (Angate, 1972), l'épiploon et le colon étaient les viscères les plus fréquemment retrouvés. Les risques de nécrose du contenu du sac herniaire sont proportionnels aux degrés et à la durée de compression des vaisseaux mésentériques. La plastie aponévrotique est la technique opératoire habituelle, surtout pour les hernies ombilicales à petit ou moyen collet (Ngom 2015) .

\section{Aspects évolutifs}

Le nombre de suppuration pariétale assez élevé dans notre travail pourrait s'expliquer par des conditions de travail encore précaires dans les pays sous-développés. Ces chiffres restent très élevés par rapport à l'étude de Ngom ( Ngom, 2015) où un seul cas de suppuration pariétale a été noté alors que dans le travail de Fall (Fall 2006)on notait une suppuration pariétale chez deux patients.

\section{Conclusion}

La hernie ombilicale chez l'enfant est une pathologie ubiquitaire dans les pays en voie de développement potentiellement grave par ses complications. Son étranglement est une réalité, mettant parfois en jeu le pronostic vital de l'enfant. L'absence de toute possibilité de surveillance active des hernies de petit collet et les épisodes d'engouement imposent un traitement chirurgical des hernies ombilicales de l'enfant. 


\section{Déclaration de conflits d'intérêt} travail.

Les auteurs ne déclarent aucun conflit d'intérêt concernant à ce

\section{Remerciements}

Nous remercions tous le personnel du service de chirurgie du centre hospitalier régional de Ziguinchor. Mention spéciale au Pr G Ngom pour avoir accepté de corriger la dernière version de ce manuscrit

\section{References:}

1. Weik J., Moore D.. An usual case of umbilical hernia rupture with evisceration. Pediatr. Surg, 2005; 33-35.

2. Stovroff M. Umbilical problems. In: Glick P, Pearl R, Irish M, Caty $M$ (eds) Pediatric surgery secrets, Hanley Belfus INC, Philadelphia, 2001; 85-87

3. Ameh E.A., Chirdan L. B., Nmadu P.T., Yusufu L.M. Complicated umbilical hernias in children. Pediatr Surg Int 2003; 19, 280-282

4. Ngom G., Gassama F., Kane A., Seck M., Ndour O., Ndoye M. Clinical and surgical aspects of strangulated umbilical hernias in children: a prospective study of 35 cases Journal of Pediatric Surgical Specialties, 2015; 9 (2), $51-52$

5. Koura A., Ogouyemi A., Hounnou G.M., Agossou-Voyeme A.K., Goudote E. Les hernies ombilicales étranglées chez l'enfant au C.N.H.U. de Cotonou : à propos de 111 cas. Méd Afr noire, 1996; (12) 43

6. Brown R. A., Numanoglu A., Rode H. Complicated umbilical hernia in Childhood. S Afr J Surg ,2006; 44:136-137

7. Lassaletta L., Fonkalsrud W. ,Tovar J. A., Dudjeon D., Asch M. J. The management of umbilical hernias in infancy and childhood. $\mathrm{J}$ Pediatr Surg, 1975; 10, 405-409.

8. Fall I., Sanou A., Ngom G., Dieng M., Ndoye M. Strangulated umbilical hernias in children. Pediatr Surg Int, 2006; 22, 233-235.

9. Khen-Dunlop N., Audry G. Management of umbilical hernia in children. Journal de pédiatrie et de puériculture , 2007 ; 20, 78-80

10. Mawukpe Vovor V., Odoulalu H., Goudote E. Les occlusions intestinales mécaniques à Dakar (à propos de 443 observations). Bull. Soc. Méd. Afr. Noire Lgue Française, 1971 ; 16, 635-643.

11. Harbans S.S., Indemohan S.N. Patterns of external abdominal hernia an analysis of 1600 consécutives cases. Indian Journal of Médical Science., 1972; 26, 706-709.

12. Miller R.H. Umbilical hernia. N. Engl. J. Méd.,1932; 206, 389-391. 
13. Mestel A.L., Burns H. Incarcerated and strangulated umbilical hernias. Clinical pediatrics, $1963 ; 2$ (2), 368-370.

14. Ndoma Ngatchoukpo V., Gaudeuille A. Kpidou-line M., Bobossi Serengbe G. Hernie ombilicale de l'enfant : aspects épidémiologiques, cliniques et thérapeutiques au complexe pédiatrique de Bangui (RCA). Rev Cames Santé , 2013 ; 1 (1),8-10 SAN

15. Bandré E. Kaboré R.A.F., Sanou A. I. Ouédraogo · O. Soré · T. Tapsoba · B. Nébié · A. Wandaogo · B. Bachy Hernies ombilicales étranglées chez l'enfant au Burkina Faso : différences avec les pays développés. Bull. Soc. Pathol. Exots $2010 ; 103,100-103$.

16. Angate Y., Kekeh J.K., Coulibaly A.O. (Statistique hospitalière de 478 hernies étranglées opérées en 4 ans au C.H.U. de Treichville. Annales de l'Université d'Abidjan, 1972; 6: 109-119.

17. Liberek V., Wildhaber B. () Strangulated umbilical hernia: Really so rare? Arch Pediatr2013; 20, 89-93

18. Ngom G., Gassama F., Mohamed A. S., Ndour O. Umbilical hernia in children in a developing country: Does the season have an effect on the occurrence of strangulation? S Afr Med J, 2016; 106 (8):744.

19. Wade T, Ba P, Ngom A, Diop B, Diao M, Konaté I. Strangulated Umbilical Littre's Hernia In A Paediatric Patient: A Case Report. The Internet Journal of Surgery. 2015; 33 DOI: 10.5580/IJS.30920

20. John J. S. Martis., Rajeshwara K. V., Kalpana Shridhar M., Deepak Janardhanan, Sunil Sudarshan. Strangulated Richter's Umbilical Hernia - A Case Report. Indian J Surg; 2011; 73(6), 455-457. 